\title{
Could Compression Be of General Use? Evaluating Memory Compression across Domains
}

\author{
SOMAYEH SARDASHTI and DAVID A. WOOD, University of Wisconsin-Madison
}

Recent proposals present compression as a cost-effective technique to increase cache and memory capacity and bandwidth. While these proposals show potentials of compression, there are several open questions to adopt these proposals in real systems including the following: (1) Do these techniques work for real-world workloads running for long time? (2) Which application domains would potentially benefit the most from compression? (3) At which level of memory hierarchy should we apply compression: caches, main memory, or both?

In this article, our goal is to shed light on some main questions on applicability of compression. We evaluate compression in the memory hierarchy for selected examples from different application classes. We analyze real applications with real data and complete runs of several benchmarks. While simulators provide a pretty accurate framework to study potential performance/energy impacts of ideas, they mostly limit us to a small range of workloads with short runtimes. To enable studying real workloads, we introduce a fast and simple methodology to get samples of memory and cache contents of a real machine (a desktop or a server). Compared to a cycle-accurate simulator, our methodology allows us to study real workloads as well as benchmarks. Our toolset is not a replacement for simulators but mostly complements them. While we can use a simulator to measure performance/energy impact of a particular compression proposal, here with our methodology we can study the potentials with long running workloads in early stages of the design.

Using our toolset, we evaluate a collection of workloads from different domains, such as a web server of CS department of UW-Madison for 24h, Google Chrome (watching a 1h-long movie on YouTube), and Linux games (playing for about an hour). We also use several benchmarks from different domains, including SPEC, mobile, and big data. We run these benchmarks to completion.

Using these workloads and our toolset, we analyze different compression properties for both real applications and benchmarks. We focus on eight main hypotheses on compression, derived from previous work on compression. These properties (Table 2) act as foundation of several proposals on compression, so performance of those proposals depends very much on these basic properties.

Overall, our results suggest that compression could be of general use both in main memory and caches. On average, the compression ratio is $\geq 2$ for $64 \%$ and $54 \%$ of workloads, respectively, for memory and cache data. Our evaluation indicates significant potential for both cache and memory compression, with higher compressibility in memory due to abundance of zero blocks. Among application domains we studied, servers show on average the highest compressibility, while our mobile benchmarks show the lowest compressibility.

For comparing benchmarks with real workloads, we show that (1) it is critical to run benchmarks to completion or considerably long runtimes to avoid biased conclusions, and (2) SPEC benchmarks are good representative of real Desktop applications in terms of compressibility of their datasets. However, this does not hold for all compression properties. For example, SPEC benchmarks have much better compression locality (i.e., neighboring blocks have similar compressibility) than real workloads. Thus, it is critical for designers to consider wider range of workloads, including real applications, to evaluate their compression techniques.

Authors' addresses: S. Sardashti and D. A. Wood, Department of Computer Sciences, University of Wisconsin-Madison, 1210 West Dayton Street, Madison, WI 53706-1685, USA.

Permission to make digital or hard copies of all or part of this work for personal or classroom use is granted without fee provided that copies are not made or distributed for profit or commercial advantage and that copies bear this notice and the full citation on the first page. Copyrights for components of this work owned by others than ACM must be honored. Abstracting with credit is permitted. To copy otherwise, or republish, to post on servers or to redistribute to lists, requires prior specific permission and/or a fee. Request permissions from permissions@acm.org.

(c) 2017 ACM 1544-3566/2017/12-ART44 \$15.00

https://doi.org/10.1145/3138805 
CCS Concepts: • Computer systems organization $\rightarrow$ Architectures; Multicore architectures;

Additional Key Words and Phrases: Compression, cache and memory design, energy efficiency, performance, multi-core systems

\section{ACM Reference format:}

Somayeh Sardashti and David A. Wood. 2017. Could Compression Be of General Use? Evaluating Memory Compression across Domains. ACM Trans. Archit. Code Optim. 14, 4, Article 44 (December 2017), 24 pages. https://doi.org/10.1145/3138805

\section{INTRODUCTION}

With increasing limits on power and energy, the memory hierarchy plays an even more critical role in multi-core systems. Caches save total system energy by filtering out expensive accesses to main memory. In addition, as vendors increase the number of cores per die, there is a commensurate demand for larger on-chip caches and main memory. Using large caches and main memory is expensive. Compression has the potential to provide the benefits of larger storage at smaller costs, leading to higher performance and lower energy.

There have been several recent proposals on exploiting compression in the memory hierarchy. Software-based approaches focus on compressing pages in main memory. For example, Apple OS $\mathrm{X}$ compresses the least recently used pages to free up memory for active processes (Apple OS X Mavericks 2013). Facebook has also recently proposed Zstandard compression (Yann Collet 2016), which is optimized for compression in scale.

Hardware-based techniques span the memory hierarchy, using simpler algorithms to keep design complexity and overheads low. For example, recent proposals seek to double (or more) the effective capacity of the last level cache in a multicore processor (Pekhimenko et al. 2012; Sardashti and Wood 2013; Sardashti et al. 2014, 2016; Biswabandan and Seznec 2016). Many of studies on compression rely on workload properties that are not clear whether they would hold for large, real-world workloads. In this article, we formed a list of common hypotheses on compression properties (Table 2). These hypotheses have served as a foundation for many previous proposals. In general, we focus on confirming and analyzing general knowledge on compression and answering some open questions on compression, including the following:

(1) Does compression work for real-world workloads running for long time?

(2) Which application domains would potentially benefit the most from compression?

(3) At which level of memory hierarchy should we apply compression: cache, main memory, or both?

Our approach is to evaluate compression effectiveness in the memory hierarchy, both in main memory and caches, for a wide range of workloads. We study several real applications, including the production servers of department of Computer Sciences at University of WisconsinMadison (e.g., web, file, and database servers), memory-intensive desktop applications (e.g., Google Chrome), and gaming applications (e.g., Linux games) for up to $24 \mathrm{~h}$ on real machines. In addition, we analyze benchmarks from different classes (e.g., SPEC, mobile, and big data). For benchmarks, we run these benchmarks to completion.

To study compressibility of real applications running for long hours, it would be very hard and challenging to use simulators. Due to the complexity of existing simulators, simulations are slow. For example, the gem5 simulator (Binkert et al. 2011) with an OOO processor configuration runs a benchmark approximately 10,000 times slower compared to running it directly on a real machine. Simulating a 1 h desktop application (e.g., watching a movie on YouTube), if at all possible, 
would take over one year of simulation time. Consequently, most researchers end up evaluating their proposals with checkpoints of small benchmarks running for short runtimes on a simulator. While previous studies, such as SimPoint (Hamerly et al. 2005), have sought to identify representative points within a workload, they have focused on workload behaviors that affect the processor microarchitecture, not on data compressibility. In addition, in early design stages, when we are mostly interested in understanding data properties, we would rather avoid hassles of benchmarks and simulator development.

To address these issues, in this work, we have developed a toolset that allows us to study data in memory and caches of real machines when running our desired applications. The basic idea is that our simple tools frequently sample the contents of physical memory as well as the actual data accessed by the CPU. This enables us to evaluate the compressibility of data in memory and caches of essentially any workload running on a machine with the Linux operating system. With our low-overhead technique, we are able to study workloads running for much longer execution times than could ever be practical using simulation. Using our toolset, we study and expand several fundamental properties on compression for different application domains, providing insights for future designs.

One should note that these techniques do not replace simulators but mostly complement them. While simulators can be used to carefully study many aspects of a technique, including its performance, our tools can be used earlier in design process to quickly study data properties of different applications. With our infrastructure, we can process data patterns and compression properties of data in memory and caches, rather than how compression technique can impact performance and energy.

Our key contributions include the following:

- We evaluate compression using selected examples from different applications. We use real applications running with real data on real machines, as well as several benchmarks running to completion.

- We study compressibility at both main memory and caches for a diverse set of workloads from different applications domains, including servers, mobile, desktop, games, and big data.

- We have developed two new tools that enable studying compressibility on real machines. These tools enable studies on cache and memory compression for much longer execution times than simulation.

- We study and analyze several fundamental properties on compression, such as compression locality. These properties are fundamental for previously proposed designs.

Using our tools, we find the several insights on compression:

- In terms of using benchmarks versus real applications, we show that it is important to run benchmarks for long runtime or to completion to avoid biased conclusions. When running to completion, SPEC benchmarks are good representative of real desktop applications in terms of compressibility of their datasets. However, this does not hold for all compression properties. For example, SPEC benchmarks have much better compression locality (i.e., neighboring blocks have similar compressibility) than real workloads. Thus, it is critical for designers to consider wider range of workloads, including real applications, to evaluate their compression techniques.

- Compression could be of general use both in caches and main memory. On average, we observe the compression ratio of $\sim 2.7$ and $\sim 2.0$, respectively, in main memory and caches; $64 \%$ and $54 \%$ of our workloads are compressible by at least a factor of two, respectively, in main memory and caches. 
- Memory data are generally more compressible than cache data due to a higher number of zero blocks in memory (i.e., on average $18 \%$ ) than in caches $(\sim 1 \%)$.

- Workloads from different domains exhibit different compressibility. Servers have the highest compression ratio (7.6) while mobile benchmarks exhibit the lowest compression ratio (1.8).

The article is organized as follows. We explain our infrastructure, tools, and workloads in Section 2. We discuss compression effective in the memory hierarchy in Section 3. Finally, Section 4 concludes the article.

\section{INFRASTRUCTURE}

\subsection{Toolsets}

Simulators are appropriate tools for studying performance and energy impacts of new features. However, they limit the breadth and length of studies when designers want to analyze wide range of workloads over long period of time. Our goal in this study is to look at a much broader range of platforms, with complete runs or long runs of live workloads. We are focusing on analyzing data compressibility, not on measuring performance or energy.

To enable studying compression on real machines, we have developed two sampling-based tools: memory compression tool and cache compression tool. These tools provide fast access to data stored in caches and main memory on a real machine. They enable us to analyze data of actively used production servers for $24 \mathrm{~h}$, without interrupting the server's daily use. Such a study would require more than 24 years of simulation on gem5. In addition, creating representative checkpoints for servers, such as mail servers, is hard. Our tools can measure essentially any running workload. Other than compression, these tools also enable studies on workload properties regarding to data in memory or caches. These tools and collected data will be public soon (we are cleaning the code and making it ready for the release on github).

2.1.1 Memory Compression Tool. The basic idea of our memory compression tool is to periodically take snapshots of the physical memory of a running workload. Our tool does this using the Linux proc filesystem and the Linux ptrace system call to analyze data in main memory. Linux ptrace (process trace) is a system call that one process can use to control another process. For example, a process can use ptrace to stop a running process and read a particular memory address of that process. Linux proc pseudo-filesystem (/proc) provides an interface to kernel data structures. For example, for a process, Linux proc pagemap (/proc/pid/pagemap) allows userspace programs to examine the page tables and related information of the given process. Similarly, Linux proc maps (/proc/id/maps) provide information on memory regions (e.g., stack or heap) of a process, including their virtual addresses.

In our tool, for a running target workload, we use Linux ptrace to periodically stop a running workload, read and analyze its pages in main memory. To find the address of blocks of the workload in memory, we use Linux map and pagemap interfaces (/proc/id/maps and /proc/id/pagemaps). Overall, our tool takes the following steps to take a snapshot of memory footprint of a running workload:

Step 1: For a running process with a given process id (pid), our tool finds its virtual memory regions by parsing its virtual memory region mapping found through "/proc/pid/maps." For each virtual memory region, this file includes the start and end addresses along with region descriptions (e.g., heap or stack). 
Step 2: For each page in a given virtual memory region (found in step 1), we then parse its pagemap "/proc/pid/pagemap" to find whether the page is present in the physical memory, and if so, to get its physical address.

Step 3: For the physical pages found in step 2, we then read their contents through ptrace interface and calculate their compressibility. We repeat step 2 and step 3 for all pages in each region.

A running workload on Linux relates to one parent process and maybe multiple spawned processes. In case there are more than one process (e.g., for servers and multi-threaded workloads), we repeat these steps for each process. Our tool also handles synonyms and does not double count physical pages mapped by more than one virtual page. Unless otherwise stated, the page size is $4096 \mathrm{~B}$, and the cache block size is $64 \mathrm{~B}$.

For a workload, we repeat this procedure (steps 1-3) periodically. With our tool, you can define how often you want a sample. For benchmarks, we take samples every few seconds until the benchmark completes. For long running servers, we take a snapshot every half an hour. For real desktop applications, we take snapshots every few minutes. A copy of this tool can be downloaded from: http://pages.cs.wisc.edu/ somayeh/memory_compression_tool.tgz.

2.1.2 Cache Compression Tool. Unlike memory, there is no direct way to scan cache contents in a real machine for a specific running workload. Thus, it is more challenging to get a sample of data in caches in real machines. To find the compressibility of cached data in a real system, we build on BadgerTrap (Gandhi et al. 2014), a tool that enables instrumentation of x86-64 TLB misses. BadgerTrap allows tracing data TLB misses in a Linux machine for a running workload. It does so by converting hardware-assisted page walks to page faults handled by a special softwareassisted TLB handler. In its current form, BadgerTrap counts the number of TLB misses. Our basic approach is to use BadgerTrap to get a random sample of data blocks accessed by CPUs for a running workload. We use BadgerTrap to find blocks accessed by CPUs, since with BadgerTrap, we know what caused data TLB misses. BadgerTrap enables us to monitor these TLB misses. We have changed the TLB miss handler of BadgerTrap so we read the data block caused a data TLB miss. Although data blocks could bypass the caches, modern processors store most accessed blocks in on-chip caches. Thus, this accessed data block, which caused the first TLB miss after a flush, will be stored in the on-chip caches. Overall, using this technique, by capturing randomly accessed data blocks, we can represent the data blocks randomly accessed at L1 data caches on a real machine.

To get a random unbiased sample of blocks, we (1) force data TLB flushes, (2) find which address caused the first TLB miss right after the flush, and (3) read that data block and measure its compressibility. To force frequent TLB flushes, we change the Linux kernel and force a data TLB flush in the Linux timer interrupt handler. Thus, while running a workload, the system will get TLB flushes periodically. To have an unbiased sample of data blocks, right after each TLB flush, we capture only the first TLB miss. This TLB miss is due to a CPU accessing a data block. In our setup, a timer interrupt occurs every $1 \mathrm{~ms}$ (frequency of $1,000 \mathrm{~Hz}$ ). Thus, we measure compressibility of a block accessed by CPU every $1 \mathrm{~ms}$. Therefore, for a small workload running for only $15 \mathrm{~min}$, we capture 900,000 blocks. With a $32 \mathrm{~KB}$ L1 cache, which holds 512 blocks, 900,000 blocks equate scanning the whole L1 cache for 1757 times. Therefore, although with our methodology, we are sampling the cache, we capture several samples as we run workloads for long runtime (up to $1 \mathrm{~h}$ ). In our desktop machine with this kernel change, we then run our workloads using BadgerTrap.

Using BadgerTrap, we can get samples of accesses to data blocks but not instruction blocks. As instructions are read-only, their compressibility in main memory should be similar to their compressibility in caches. Also, as this tool involves modifying Linux kernel, we use it on our 
desktop machine to measure cache compressibility of real desktop applications and benchmarks. It is not feasible for us to use it on our production servers.

2.1.3 Strengths and Limits of These Tools. Our main goal of developing these tools is to enable us to analyze data compressibility of real workloads. Using these tools, one can sample blocks in main memory or caches of a real machine. This way, a designer can ignore hassles of benchmarking and simulation for early stages of designs when studying potentials or validating some hypotheses.

However, these methodologies cannot replace simulators. Although we can use these tools to study data in memory and caches, we cannot use them to evaluate performance or energy impacts. As discussed in previous work (Sardashti and Wood 2013), higher compressibility does necessarily translate to higher performance. There are many other factors in design, in addition to workload properties, such as sensitivity of cache size and footprint size. To do such analysis, one should implement those ideas in a cycle-accurate simulator or in a hardware platform.

Since our techniques are based on sampling, like any other sampling technique, sampling frequency would impact the outcome. For our memory compression tool, we tuned the sampling frequency based on total execution of time of the workload. For cache compression tool, we take a sample on a timer interrupt handler (every $1 \mathrm{~ms}$ in our system). Thus, picking the right frequency would be important.

2.1.4 Validating Cache Compression Tool. As we discussed earlier in this section, on every timer interrupt (every 1ms), the cache compression tool flushes the data TLB first. It then measures the compressibility of the very first block that missed in TLB. Since blocks accessed by CPUs are mostly cached in L1 data caches, we used these samples to measure compressibility of cached data blocks in a real system.

To validate our tool, we used a gem5 simulator (Binkert et al. 2011) with 32KB L1 caches (similar to our desktop machine). We ran 13 SPEC benchmarks to completion. We measure compressibility of blocks accessed at L1 data caches. For each benchmark, we measure the average compression ratio of all accessed blocks from L1 data caches, as well as compressibility of blocks sampled every $1 \mathrm{~ms}$ (similarly to our tool).

Table 1 summarizes these results Compression ratio measured by our tool is very close to what we measured by sampling compressibility of accessed cache blocks at data L1 caches in gem 5 every $1 \mathrm{~ms}$. Compared to compressibility of all accessed blocks, our cache tool measured a compression ratio of about 0.1 higher than gem 5 for all blocks. In another word, our cache tool measures average compressed block size of 34.05 bytes, while gem 5 measures an average compressed size of 35.79 bytes for all accessed blocks in L1 data caches. This translates to a 1.75-byte difference (we consider 64B cache block sizes).

Overall, these analysis shows compression ratio measured by our cache tool is on average $0.3 \%$ and up to $17 \%$ (1.34 vs. 1.14 for sphinx) different from what we measure for all accessed blocks by gem5.

\subsection{Platforms}

We run our workloads on two platforms:

Servers: In this work, we evaluate compressibility of three servers in production use in a university department: a webserver, a fileserver, and a Postgres database server. All these servers run RedHat Enterprise Linux 6.5. The fileserver and the Postgres server run on a machine with 2 Intel Xeon cores, while the webserver run on a four-core Intel Xeon machine.

Desktop Machine: We also evaluate several real desktop applications and benchmarks. We run those on a desktop machine with Ubuntu 13.10. The machine has 4 Intel Corei5-2500K cores. 
Table 1. Compression Ratio Measured by Gem5 vs. Our Cache Compression Tool

\begin{tabular}{|l|c|c|c|}
\hline Application & $\begin{array}{c}\text { Our Cache } \\
\text { Compression } \\
\text { Tool }\end{array}$ & $\begin{array}{c}\text { All accessed } \\
\text { L1 data blocks } \\
\text { by gem5 }\end{array}$ & $\begin{array}{c}\text { L1 data blocks } \\
\text { accessed every } \\
\text { 1ms by gem5 }\end{array}$ \\
\hline Astar & 2.06 & 2.17 & 2.04 \\
\hline Gcc & 2.80 & 3.19 & 2.86 \\
\hline h264ref & 1.80 & 1.67 & 1.81 \\
\hline hmmer & 1.54 & 1.67 & 1.56 \\
\hline libquantum & 2.35 & 2.36 & 2.36 \\
\hline Mcf & 2.02 & 2.13 & 2.23 \\
\hline Milc & 1.23 & 1.06 & 1.09 \\
\hline namd & 1.20 & 1.12 & 1.19 \\
\hline omnetpp & 2.12 & 2.21 & 2.18 \\
\hline povray & 1.84 & 1.78 & 1.86 \\
\hline soplex & 1.99 & 2.17 & 2.08 \\
\hline sphinx & 1.34 & 1.14 & 1.10 \\
\hline geomean & $\mathbf{1 . 8 0}$ & $\mathbf{1 . 7 9}$ & $\mathbf{1 . 7 8}$ \\
\hline
\end{tabular}

\subsection{Compression Algorithms}

There are several compression algorithms. Using our tools, we can simply model and use any algorithm. Here we study four representative compression algorithms. There are several other algorithms that one could pick for these studies (Arelakis and Stenstrom 2014; Arelakis; Biswabandan and Seznec 2016). Here, we pick three most popular ones. The first three algorithms have been widely used to study compressed cache and memory designs:

C-PACK + Z: Cache Packer (C-PACK) (Chen et al. 2010) is a lossless compression algorithm that is designed specifically for hardware-based cache compression. C-Pack compresses a data block at a 4-byte word granularity. It detects and compresses frequently appearing words (such as sign-extended words or zero words) to fewer bits. In addition, it also uses a small dictionary to compress other frequently appearing patterns. The dictionary has 16 entries, each storing a 4-byte word. The dictionary is built and updated per data block. C-PACK checks whether each word of the input block would match a dictionary entry (even partially). If so, C-PACK then stores the index to that entry in the output compressed code. Otherwise, C-PACK inserts the word in the dictionary. C-PACK takes 16 cycles to compress a 64-byte data block, and nine cycles to decompress at $3.2 \mathrm{GHz}$ (Sardashti and Wood 2013; Chen et al. 2010). In this work, we use a modified version of C-PACK $(C$ PACK $+Z)$ that also detects zero blocks (Sardashti and Wood 2013).

FPC + Z: Frequent Pattern Compression (FPC) is a significance-based compression algorithm (Alameldeen and Wood 2004). It exploits the fact that many values are small (e.g., small integers) and do not require the full space allocated for them. FPC compresses data blocks on a word-byword basis by storing common word patterns (such as sign-extended words or repeated bytes) in a compressed format accompanied with an appropriate prefix. Compared to dictionary-based approaches, FPC has lower decompression latency. FPC decompresses a 64-byte line in five cycles, assuming 12 FO4 gate delays per cycle. We also augment FPC to detect zero blocks $(\mathrm{FPC}+\mathrm{Z})$.

BDI: Base-Delta-Immediate (BDI) compression algorithm (Pekhimenko et al. 2012) is another low-overhead algorithm optimized for cache/memory compression. It is based on the observation that in a cache/memory block, many words have small differences in their values. BDI encodes a 
block as one or more base-values and an array of differences from the base-values or simply zero. In this work, we use a version of BDI that is optimized for capacity (i.e., high compression ratio). It uses two base values and takes two to three cycles to decompress a block.

gzip: To estimate the potential of compression with a complex algorithm, we use gzip. gzip is based on the DEFLATE algorithm, which is a combination of LZ77 and Huffman coding. We run gzip with its highest compressibility level (i.e., gzip-9). This algorithm is too complex for hardwarebased compressed caches or memory. However, gzip can give us an approximate bound on compressibility of our workloads when compressing large chunks of memory. However, gzip is not a real bound for all data types including floating point as it is not optimized for those.

For all these algorithms, we compress 64-byte blocks unless otherwise mentioned. We use $\mathrm{C}-\mathrm{PACK}+\mathrm{Z}$ in most experiments as a representative of hardware-based algorithms, as it is shown to have a good compressibility with low overheads (Sardashti and Wood 2013).

\subsection{Workloads}

Since compression is a candidate in many platforms including servers, desktops, mobiles, games, and big data, we consider a suite of workloads that span these.

Servers: We analyze three servers providing production service: a webserver, an AFS fileserver, and a Postgres database server used for Linux backup metadata. These servers are actively used in our department. We analyze these servers for $24 \mathrm{~h}$.

Real Desktop Applications: We use five real desktop applications running on our desktop machine for about $1 \mathrm{~h}$. We use Google Chrome while streaming a one-hour long movie on YouTube, Firefox while browsing a Wikipedia page (i.e., wiki) with text and pictures (http://en.wikipedia. org/wiki/United_States), gedit text editor while editing a large text file, Open Office Writer (i.e., openWrt) while editing a version of this article, and Open Office Calc (openCalc) while editing an excel file.

Gaming: We run three games supported on Ubuntu: Battle for Wesnoth (Wesnoth), Extreme Tux Racer (Tracer), and Pingus. We play each game for about an hour on our desktop platform.

Desktop Benchmarks: In addition to real desktop applications, we use SPEC CPU2006 suite with reference input sets. We use astar, bzip2, gcc, gobmk, h264, hmmer, libquantum, mcf, omnetpp, perlbench, and sjeng from SPEC-CINT, in addition to milc, namd, povray, soplex, and sphinx from SPEC-CFP.

Mobile Client: We use CoreMark (Coremark) for ten million iterations, which is a widely used benchmark for evaluating mobile systems. We also use BBench (Gutierrez et al. 2011), a web-page rendering benchmark.

Big Data: We use graph-analytics and memcached from Cloudsuite (Ferdman et al. 2012) and Graph500 [Murphy et al. 2010] as examples of emerging big data workloads. Memcached simulates the behavior of a Twitter caching server. Graph analytics runs a machine learning and data mining software. We run them with default parameters (Cloudsuite). Graph500 generates a large graph, compresses it into sparse structures, and then does parallel breadth-first search. We use it with a scaling factor of 24 (8GB memory footprint).

Similar to previous work, we also use some popular benchmarks; however, we run these benchmarks to completion. Figure 1 indicates the importance of running benchmarks for long runtime. Figure 1 shows the compression ratio (original size/compressed size) of cached blocks accessed for two SPEC benchmarks over a fraction of their execution time. For these experiments, we use our cache compression tool. Each point in these graphs shows the average compression ratio for 1,000 consecutive randomly accessed cache blocks.

We can infer two important points from these graphs: (1) compression ratio changes over time (2) in different phases, a different algorithm might perform the best. Here, we showed only 
(a)
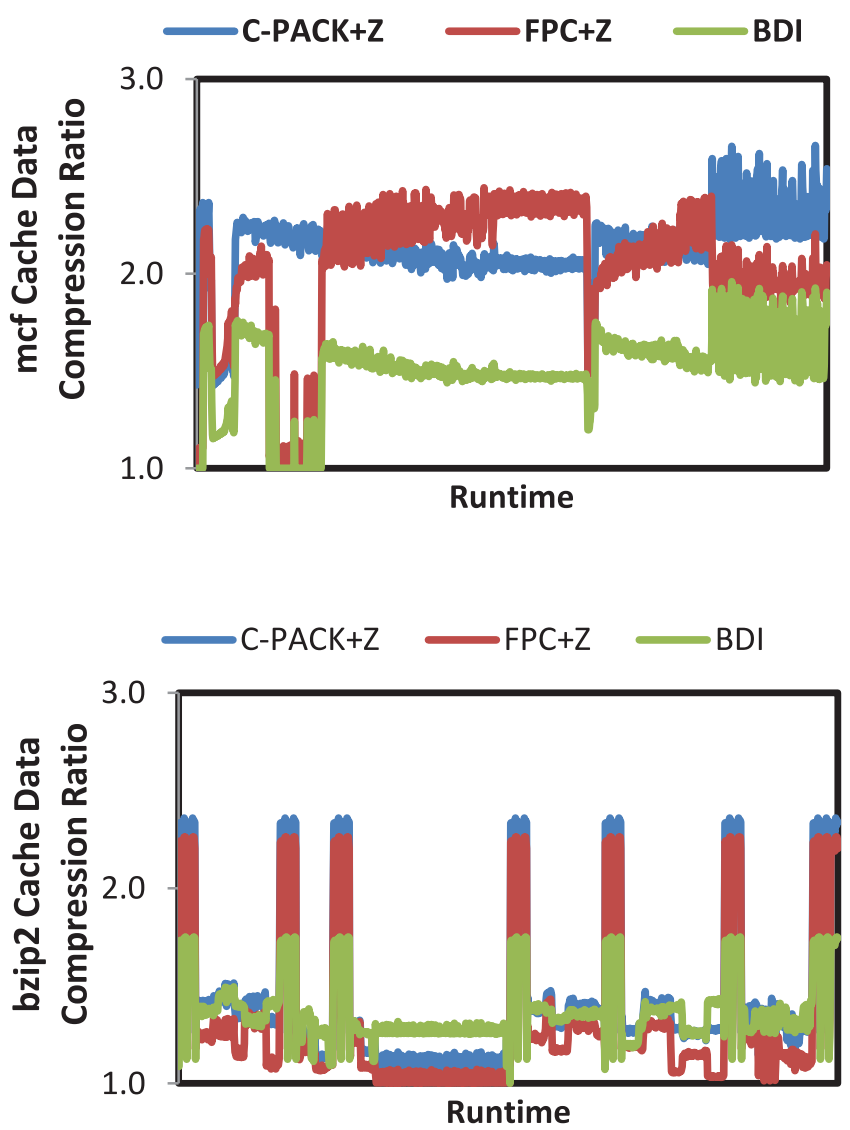

(b)

Fig. 1. Compression ratio of data blocks, each point shows compression ratio of 1,000 consecutive cache accesses over runtime for mcf (a) and bzip2 (b).

two examples, but other benchmarks have similar behaviors too. For example, with C-PACK $+\mathrm{Z}$ algorithm, compression ratio of mcf changes from about 1.5 to 2.5 over time. For mcf, in some periods, $\mathrm{C}-\mathrm{PACK}+\mathrm{Z}$ gains the most compressibility, and in some others, $\mathrm{FPC}+\mathrm{Z}$ does so. We see similar pattern for bzip2. Compressibility of bzip2 changes from almost 1 (not compressible) to 2.5 for the shown snapshot.

The main takeaway is as follows: When running small benchmarks, it is critical to run them to completion (or for long runtime). If one study wants to find which algorithm suites the best for a benchmark, then even using a technique such as SimPoint (Hamerly et al. 2005) might not help. Therefore, in this article, whenever we evaluate benchmarks, we run them to completion.

\section{COMPRESSION EFFECTIVENESS IN MEMORY HIERARCHY}

There have been many proposals on exploiting compression to improve cache and memory utilization. Many of these studies rely on workload properties that have only been demonstrated to hold for small samples of benchmarks with mostly synthetic data. In this work, we treat these workload properties as hypotheses that must be tested, listed in Table 2. A compressed cache or main memory scheme has two main parts: a compression algorithm to represent a block's information with fewer bits and a compaction mechanism to fit compressed blocks in the cache 
Table 2. Hypotheses on Compression

\begin{tabular}{|l|}
\hline 1. Most workloads are compressible: $50 \%$ of workloads have compression ratio $\geq 2$. \\
\hline 2. Cache data are more compressible than memory data. \\
\hline 3. More complex compression algorithms improve compressibility. \\
\hline 4. Compression locality: neighboring blocks have similar compressibility. \\
\hline 5. Floating point data is mostly uncompressible. \\
\hline 6. Instructions are mostly uncompressible. \\
\hline 7. Bigger blocks are more compressible. \\
\hline 8. Sub-blocking eliminates most internal fragmentation. \\
\hline
\end{tabular}

or memory. The first seven properties in Table 2 focus on compressibility of workloads. The last property that we test addresses the potential for compacting compressed data. However, in this article, we focus on the effectiveness of compression algorithms, not compaction mechanisms. In the next sections, we evaluate each hypothesis using a wide range of real applications and benchmarks from different domains.

\subsection{Could Compression Be of General Use?}

Many workloads must benefit from compression to justify hardware-based compression mechanisms. We formulate this into a testable hypothesis as: at least $50 \%$ of workloads have compression ratio $\geq 2$. This is a statement of overall compression effectiveness. If this hypothesis holds, then we argue that it justifies hardware-based compression mechanisms. If this turns out to be false, then it would mean that compression might not be of general use, but that special-purpose compression schemes could still be beneficial.

To evaluate this hypothesis, we use our memory compression tool, and measure the compressibility of $64 \mathrm{~B}$ blocks in main memory using the $\mathrm{C}-\mathrm{PACK}+\mathrm{Z}$ algorithm, shown in Figure 2(a). On average, we observe the compression ratio of 2.7 across all workloads (both real application and benchmarks). Although there is a wide distribution in compressibility of these workloads, as shown in Figure 2(b), majority of workloads are well compressible. Overall, $64 \%$ of our workloads (i.e., 7 of 11 real applications and 14 of 22 benchmarks) have compression ratio $\geq 2$, verifying the first hypothesis.

Among our workloads, real applications tend to be more compressible than benchmarks with the average compression ratio of 3.7 versus 2.3, indicating high potentials of compression for realworld workloads. If we ignore the outliers in real applications, such as our fileserver with compression ratio of 18, then real applications and benchmarks have on average similar compressibility.

To better understand these results, we show the average distribution of compressed block sizes for different domains (e.g., Desktop), all workloads (ALL), and some representative workloads in Figure 3. There is huge variation across workloads. In some workloads, such as Tracer, Fileserver, and bzip2, zero blocks and highly compressed blocks are dominant in memory, resulting in high compression ratios. On the other hand, for low compressible workloads, such CoreMark and milc, many blocks are uncompressible or poorly compressible (i.e., they have large compressed block sizes). Many other workloads, such as our webserver and wiki, have more uniform distributions. Overall, on average $18 \%$ of blocks are zero, $16 \%$ of blocks are uncompressible (64B), and the rest are compressible to $1 \mathrm{~B}$ to $63 \mathrm{~B}$, resulting in good compressibility of these workloads.

\section{Which Application Domain to Compress?}

Figure 2 shows the average compression ratio of workloads from different domains, including servers, desktop, games, big data, and mobile. Workloads exhibit different compressibility. 


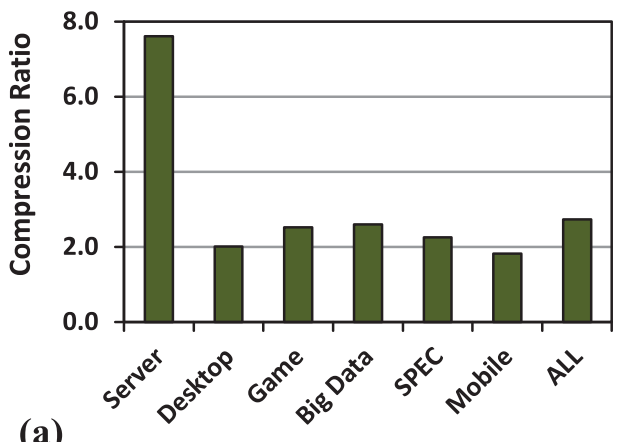

\begin{tabular}{|l|l|l|l|}
\hline & Avg & Min & Max \\
\hline Servers & 7.6 & 1.8 & 19 \\
\hline Desktop & 2.0 & 1.5 & 2.8 \\
\hline SPEC & 2.2 & 1.1 & 3.8 \\
\hline Games & 2.5 & 2.0 & 3.4 \\
\hline Big Data & 2.6 & 2.0 & 3.6 \\
\hline Mobile & 1.8 & 1.4 & 2.2 \\
\hline
\end{tabular}

(a)

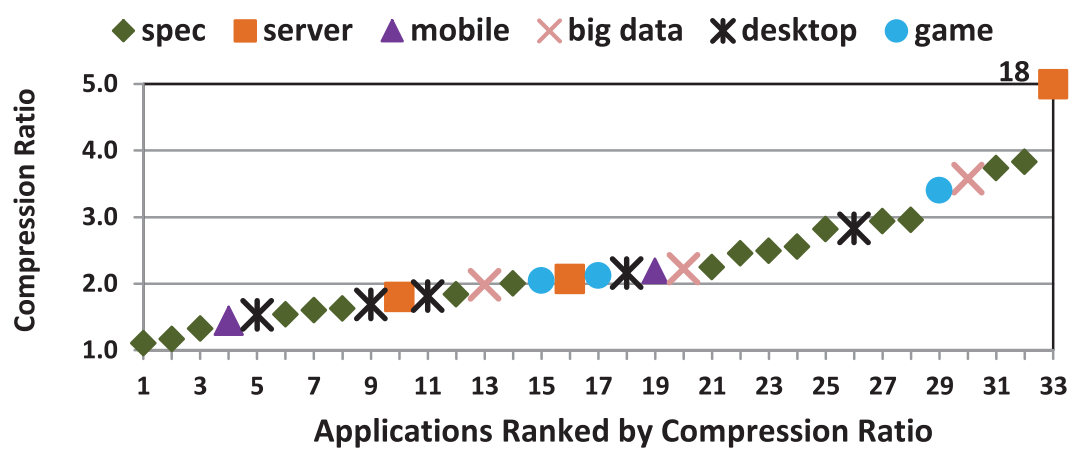

Fig. 2. ((a) and (b)) Compression ratio in memory with $\mathrm{C}-\mathrm{PACK}+\mathrm{Z}$ for the studied domains and (c) per workload.

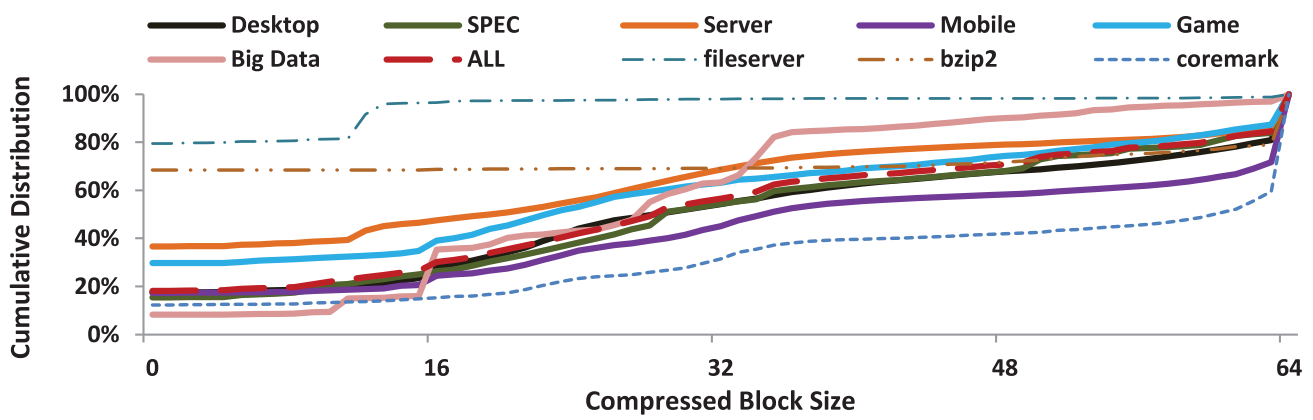

Fig. 3. Cumulative distribution of compressed block sizes in main memory.

Figure 2(b) shows the average, minimum, and maximum compression ratio measured for each workload domain. Among our workloads, Servers show on average the highest compressibility with an average of 7.6, while our mobile benchmarks show the lowest compressibility. Desktop applications, as well as SPEC benchmarks, also experience a wide range of compressibility. For big data applications and games, the results are more stable, with all these workloads having a minimum compressibility of 2.0.

Servers: Among our workloads, fileserver shows the highest compressibility with compression ratio of about 18. Our other server workloads, Postgress and webserver, also show good compression ratio (an average of 2 ). 
To better understand these results, Figure 3 shows the distribution of compressed block sizes. In our servers, on average $70 \%$ of blocks are compressible to less than half (32 bytes). For these servers, on average, about $40 \%$ are zero blocks. For our fileserver, in particular, $80 \%$ of blocks are zero, resulting to its high compression ratio. These many zero blocks are mostly due to zero padding of pages. Even, ignoring zero blocks, majority of blocks in servers are compressible, with only $13 \%$ uncompressible blocks overall.

Desktop Workloads: For desktop workloads, we observe a mix of compressibility. Desktop applications have an average compression ratio of 2.0, with the maximum compression ratio of 2.8 for openWrt, and the lowest compression ratio of 1.5 for gedit. For Desktop workloads, as shown in Figure 3, about $54 \%$ of blocks are compressible to half or less ( 0 to 32B). On the other hand, for gedit, with the minimum compressibility, about $30 \%$ of blocks are not compressible at all.

We observed similar pattern for SPEC benchmarks, which are usually used as representative of desktop applications. SPEC benchmarks have an average compression ratio of 2.2. As we will discuss later, SPEC benchmarks have a range of compressibility too.

Overall, based on these analyses, desktop workloads would be a good target for adaptive compression techniques, where we enable compression for those with potentials (Alameldeen and Wood 2004).

Games: We evaluated compressibility of three games supported on Ubuntu, while playing them for about an hour. We observe good compression ratio for these workloads with an average compression of 2.5. As shown in Figure 3, on average more than $60 \%$ of memory blocks of these workloads are compressible to zero to 32B. For etracer, which has the highest compression ratio, we observed significant number of zeros in memory (about $60 \%$ of blocks).

Big Data Workloads: Similarly, we analyzed the compressibility of our big data workloads. Big data workloads have on average compression of 2.6. Interestingly, for these workloads, many of their blocks are compressible to $16 \mathrm{~B}$ or $32 \mathrm{~B}$. For these workloads, there are still about $8 \%$ of blocks which are zero, but about half of the blocks were compressed to $16 \mathrm{~B}-32 \mathrm{~B}$. This shows high potentials of compression this domain.

Mobile Workloads: Among benchmarks, we observe the lowest compressibility for mobile benchmarks (on average 1.8). The memory footprint of these benchmarks has many uncompressible blocks. Note that workloads with low compressibility might still benefit from special-purpose compression algorithms (Ratanaworabhan et al. 2006; Burtscher et al. 2007, 2010).

\section{Is SPEC Representative of Real Desktop Applications?}

Among benchmarks, SPEC benchmarks have been frequently used to evaluate compression techniques to represent desktop application domain. Thus, here we compare them with our set of real desktop applications. As we explained earlier in this section, studying only one short snapshot of these benchmarks (e.g., running a checkpoint for few thousand CPU cycles in a simulator) could mislead the designers. Thus, unlike most previous studies, we run these benchmarks to completion.

Similarly to the real desktop applications (Desktop in Figure 3), we observe a mix of compressibility levels for these benchmarks, with the maximum of 3.8 for bzip2, the minimum of 1.1 for milc, and the average compression ratio of 2.2. As we show in Figure 3, for bzip2, about $68 \%$ of its memory footprint is zero resulting to its high compression ratio. While for milc, $80 \%$ of blocks are uncompressible, explaining its low compression ratio.

On average, the distribution of compressed block sizes for SPEC benchmarks (SPEC) is also very similar to the average distribution of real desktop applications (Desktop), as shown in Figure 3. Overall, this indicates that SPEC benchmarks are representative of real desktop applications in terms of compressibility of datasets. 
Later, we show that although these benchmarks have similar compressibility as real desktop applications, their other compression properties, such as compression locality, differ significantly from real applications.

Similarly to the real desktop applications (Desktop in Figure 3), we observe a mix of compressibility levels for these benchmarks, with the maximum of 3.8 for bzip2, and the minimum of 1.1 for milc, and the average compression ratio of 2.2. As we show in Figure 3, for bzip2, about $68 \%$ of its memory footprint is zero resulting to its high compression ratio. While for milc, $80 \%$ of blocks are uncompressible, explaining its low compression ratio.

On average, the distribution of compressed block sizes for SPEC benchmarks (SPEC) is also very similar to the average distribution of real desktop applications (Desktop), as shown in Figure 3. Overall, this indicates that SPEC benchmarks are representative of real desktop applications in terms of compressibility of datasets.

Later, we show that although these benchmarks have similar compressibility as real desktop applications, their other compression properties, such as compression locality, differ significantly from real applications.

\subsection{At Which Memory Level to Compress: Caches, Main Memory, or Both?}

Several proposals have exploited compression at different levels of the memory hierarchy. However, most of them do not differentiate the impact of compression in main memory versus caches. Mahapatra et al. $(2003,2005)$ show that instructions in L1 caches have lower entropy, and so higher compressibility than instruction in main memory for their studied applications. Then, the question is whether this would hold for data blocks, too. To the best of our knowledge, there is no comparison on compressibility of data blocks in main memory vs. caches. In this section, we do this comparison that would give designers insights on where they can get the highest benefits from compression: caches or main memory?

\section{Are Caches More Compressible Than Main Memory?}

Figure 4(a) shows the average compression ratio in memory versus caches for different domains. We measure the average compression ratio of 64B blocks both in memory and in caches. Since our cache compression tool cannot analyze instructions, we evaluate compressibility of only data blocks in main memory. Here, we are not including servers, as we cannot change their Linux kernel to support our cache compression tool.

On average, our workloads have compression ratio of 2.5 for data blocks in main memory and 2.0 for data blocks in caches. Figure 4(b) shows that for all workloads cache data are not significantly more compressible than memory data. Among our workloads, 60\% (18 of 30) have similar compressibility (i.e., compression ratios less than 0.5 different) in main memory and caches. The rest of our workloads have considerably higher compression ratio in memory than L1 caches. Overall, we found that for almost all of our workloads, the compression ratio of data blocks in memory is either similar or better than the compression ratio of data blocks in the L1 data caches.

Then the question is why data blocks are more compressible in memory than caches for some of these workloads. To find out the reason, we categorize blocks in memory and caches to zero blocks and non-zero blocks. We first find the percentage of zero blocks in main memory and caches. We then compare the compression ratio for the rest of blocks (non-zero blocks). Table 3 shows the percentage of data blocks that are zero in memory versus those accessed by CPUs.

For all classes of our workloads, there are considerable numbers of zero blocks in main memory as oppose to caches. Most zero blocks in memory are due to zero padding at the end of pages and zero pages. Thus, most of these zero blocks are never read by the CPUs or placed in caches. For 
(a)

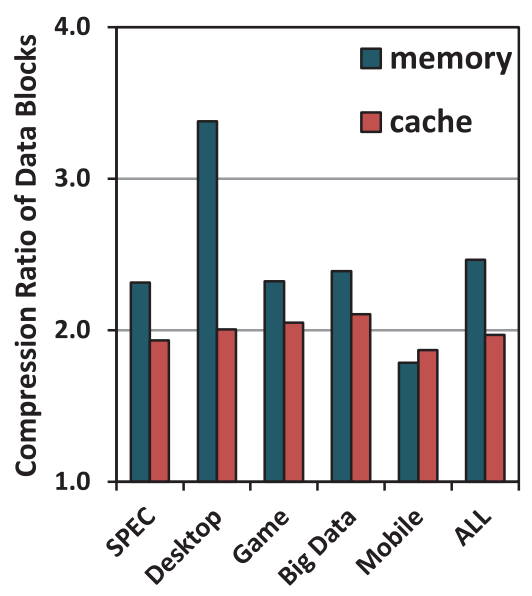

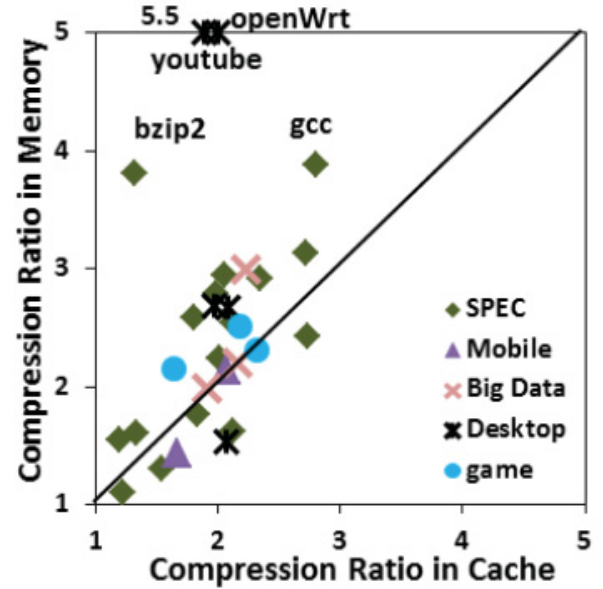

(b)

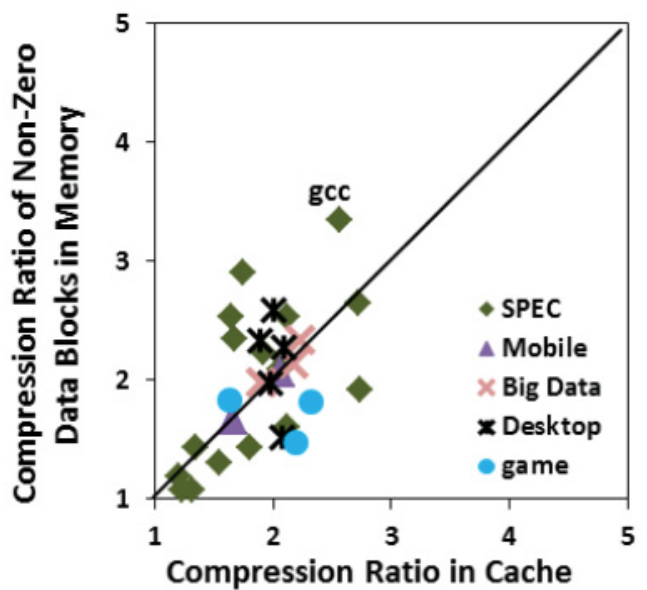

(c)

Fig. 4. Data block compression: cache vs. memory, across domain (a); per workload (b); only for NON-ZERO data blocks (c).

workloads with higher compressibility in memory than caches, the percentage of zero blocks is much higher in memory. For example, for bzip2 and OpenWrt, $35 \%$ and $36 \%$ of blocks are zero in memory, respectively. While, only about $1 \%$ of accessed blocks by CPUs are zero for these workloads.

To further check this, we also compare the compression ratio of non-zero data blocks in main memory and in caches. As shown in Figure 4(c), non-zero blocks have similar compressibility in caches and memory, with the average compression ratio of 2.0. Putting these two together, higher number of zero blocks in memory explains the lower compressibility of data in caches.

Overall, these results indicate significant potential for both cache and memory compression, with memory somewhat better due to the high incidence of zero blocks. On average, $54 \%$ of our workloads (16 of 30 ) have compression ratio $>2$ in caches, verifying that cache compression could also be of general use across different domains. 

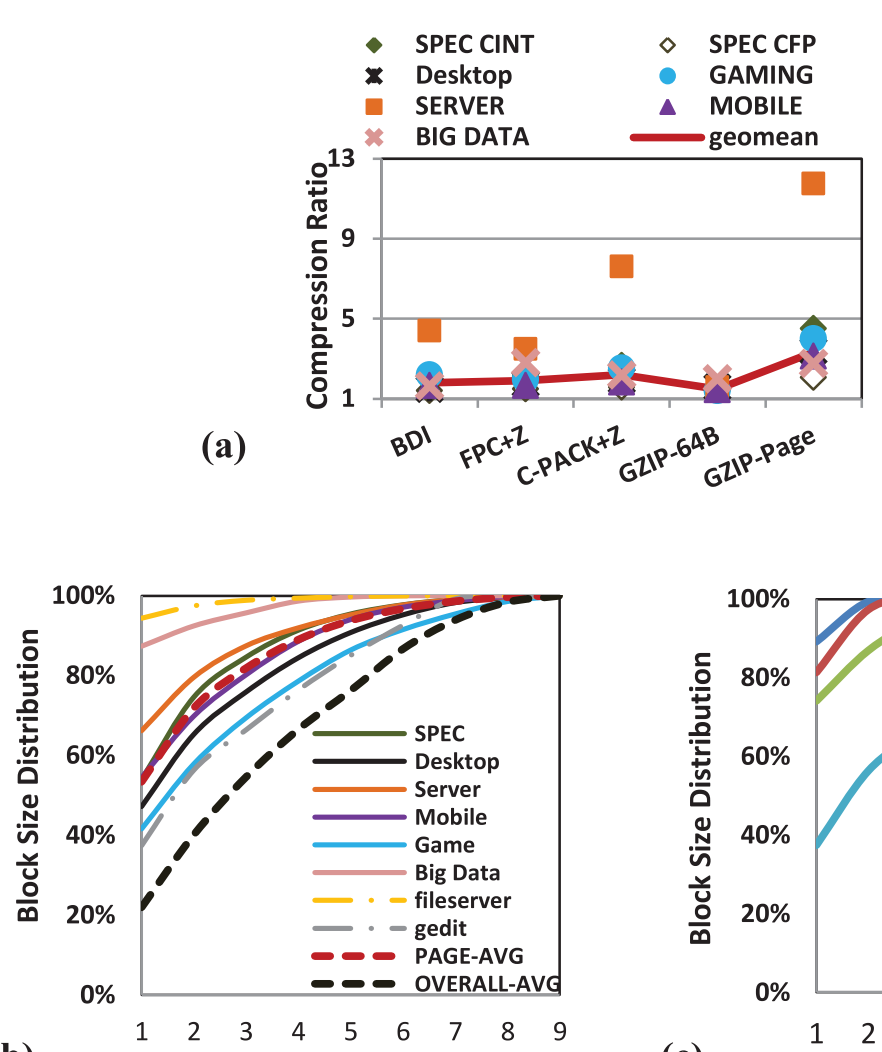

(b)

(a)

Fig. 5. (a) Memory compression with different algorithms. (b) Cumulative distribution of compressed block sizes within a page in main memory for all domains and (c) for gedit at different granularities. Sizes are ordered from the most common (1) to the least common (9) for each application on the $x$-axis.

\subsection{How Big Is the Role of Compression Algorithms?}

The compression algorithm is a critical design component in compressed caches and memory. In general, the conventional wisdom says more complex algorithms tend to improve compressibility. For a given algorithm, since its decompression latency is on the critical path, we categorize our studied algorithms based on the complexity and latency of their decompression mechanisms as follows: BDI (the simplest), FPC $+\mathrm{Z}, \mathrm{C}-\mathrm{PACK}+\mathrm{Z}$, and gzip (the most complex). In this study, we picked these four algorithms, as they have been widely used in the past.

Figure 5(a) summarizes the compression ratio of different algorithms in main memory. Despite the high complexity of gzip, it does not perform nearly as well as other algorithms for small block sizes. This holds true across different workload domains. Since gzip depends on building a dictionary of Huffman encoded strings, its effectiveness is hampered by the small block sizes used in the cache hierarchy. Thus, gzip performs the best at page granularity (GZIP-page).

At small block granularities, $\mathrm{C}-\mathrm{PACK}+\mathrm{Z}$ provides the highest compression ratio for almost all our workloads, as $\mathrm{C}-\mathrm{PACK}+\mathrm{Z}$ exploits data replication and special data patterns common in small cache/memory blocks. FPC $+\mathrm{Z}$ and BDI perform similarly on average despite the higher decompression latency of FPC $+\mathrm{Z}$. Putting these together, algorithm complexity is not always an indicator of better compressibility, rejecting the third hypothesis. 
Table 3. Percentage of Zero Blocks in Memory vs. Caches

\begin{tabular}{|l|c|c|}
\hline & $\begin{array}{c}\text { Zero data blocks in } \\
\text { memory }\end{array}$ & $\begin{array}{c}\text { Zero data blocks accessed by } \\
\text { CPUs }\end{array}$ \\
\hline SPEC & $7 \%$ & $1 \%$ \\
\hline Desktop & $13 \%$ & $1 \%$ \\
\hline Game & $15 \%$ & $1 \%$ \\
\hline Big Data & $8 \%$ & $1 \%$ \\
\hline Mobile & $16 \%$ & $0 \%$ \\
\hline Server & $21 \%$ & - \\
\hline
\end{tabular}

\subsection{Does Compression Locality Exist across Domains?}

Blocks from the same memory address space tend to have similar characteristics (Wu et al. 2011). Pekhimenko et al. (2013) extend this to compression, with a hypothesis that blocks of a page have similar compressibility (i.e., compression locality). This hypothesis has served as a foundation for low cost compressed memory and compressed cache designs. Pekhimenko et al. (2013) propose Linearly Compressed Pages (LCP) that uses a fixed size for compressed blocks within a given page of main memory to simplify lookups. Other recent works (Sardashti et al. 2014, 2016; Biswabandan and Seznec 2016) exploit compression locality within small regions (four or eight blocks) in the L3 cache, compacting neighboring blocks if possible.

These techniques (Pekhimenko et al. 2013; Sardashti et al. 2014, 2016; Biswabandan and Seznec 2016) benefit workloads that have high compression locality. Otherwise, they might lower compression benefits due to internal fragmentation. Thus, the question is whether the compression locality hypothesis holds for our diverse set of workloads.

Figure 5(b) shows the cumulative distribution of unique compressed block sizes within a $4 \mathrm{~KB}$ page. We use C-PACK $+Z$ to compress each block to 0 to 8 8-byte sub-blocks. We observed similar results using other compression algorithms and sub-block sizes. For each page, we then find the distribution of blocks ranked from the most common size to the least common size. The most common size ( 1 on the $x$-axis of Figure $5(b))$ is determined on a per-page basis. For example, for Wiki, the most common size is zero in one page and 32B in another. For each workload, we report the overall distribution. Figure 5(b) also shows the OVERALL-AVG, which is the cumulative distribution of compressed block sizes across all pages of all workloads, and PAGE-AVG, which averages the frequency of the most common size within a page across all pages and all workloads (where different pages may have different most common sizes).

\section{Does Compression Locality Exist at Page Granularity?}

In Figure 5(b), OVERALL-AVG shows that the most common compressed block size over all workloads accounts for $22 \%$ of all blocks. If compressed block sizes were randomly distributed across pages, then we would expect the per-page distribution to be similar to the overall average distribution. Instead, PAGE-AVG shows that the most common block size within a page accounts for $53 \%$ of the blocks. These results clearly show that pages exhibit compression locality, with the most common block size within a page occurring more than twice as often as the most common block size overall.

Although compression locality exists at pages, the distribution has a heavy tail and even three different block sizes only account for $82 \%$ of the blocks in a page. This suggests that compressed memory designs, such as LCP (Pekhimenko et al. 2013), that optimize for a single compressed block size per page must strike a balance. Picking too large a compressed block size increases internal fragmentation, while too small a block results in many "exceptional" blocks that do not fit. 


\section{How Does Compression Locality Change across Domains?}

Although compression locality exists, it differs per workload. In Figure 5(b), we show the average cumulative distribution for each class of workloads. Servers, big data, and SPEC show high compression locality. For example, in Servers on average 42 of 64 blocks in a page (i.e., $66 \%$ ) are compressible to the same size. On the other hand, games show the lowest compression locality at page granularity. In Pingus, the most common size accounts for only $30 \%$ of blocks in a page. On average, all nine compressed block sizes (i.e., 0-8 8-byte sub-blocks) exist in a page in Pingus.

Mobile benchmarks and real desktop application have a mix of sizes. CoreMark has high compression locality with most blocks being uncompressible, while BBench has a mix of sizes per pages. Desktop also show average compression locality, with the lowest locality for gedit, and the highest locality for OpenWrt.

These results emphasize the importance of considering a wide range of workloads. While SPEC benchmarks exhibit high compression locality, many real applications have lower locality. Therefore, these workloads might benefit less from designs exploiting compression locality (Pekhimenko et al. 2013).

\section{What Are the Most Common Compressed Block Sizes?}

The most common compressed block size changes per workloads. Over all workloads, the top three most common sizes are 64 bytes (i.e., blocks are uncompressible), zero (i.e., zero blocks), and 32 bytes (i.e., blocks are compressible to half). For workloads with lower compression ratio, such as gedit and SPEC CFP benchmarks, 64B blocks (i.e., uncompressible blocks) are the most common. On the other hand, zero blocks are the most common in highly compressible workloads, such as Fileserver.

\section{How Does Compression Locality Change at Different Granularities?}

Figure 5(c) illustrates the effect of region size on compression locality by showing the cumulative distribution of compressed block sizes for gedit. The region size decreases from 64 blocks (i.e., a page) to 4 blocks (i.e., a typical super-block size). Although gedit has low compression locality at page granularity, it has high locality for smaller regions. For example, in an 8-block region, on average 6 of 8 blocks have the same compressed size. Overall, we found that on average compression locality is higher in smaller regions. This shows the potential for designs exploiting compression locality at small granularities, such as SCC (Sardashti et al. 2014, 2016; Biswabandan and Seznec 2016).

\subsection{Compressibility of Different Data Types: Floating-Point versus Integer}

Although compression could be of general use, general-purpose compression is not necessarily appropriate for all data types. In particular, floating point data are considered mostly uncompressible using general-purpose compression algorithms (Kant et al. 2002). For all our four studied algorithms, we observed an average compression ratio less than 1.5 for SPEC CFP benchmarks.

To better understand this result, Figure 6(a) shows the percentage of integer blocks in data regions (i.e., stack and heap) in memory on the $x$-axis, and overall memory compression ratio using $\mathrm{C}$-PACK $+\mathrm{Z}$ on the $y$-axis. Similarly to Kant et al. (2002), we classify blocks as integer if the $8 \mathrm{MSBs}$ are $0 \times 00$ or $0 x F F$ in each 32-bit word. In this way, we detect small integer values, which are most common. Since C-PACK $+Z$, similarly to other basic algorithms, is mostly optimized for small integer values, the higher the percentage of non-integer blocks (including floating-point data), the lower the compression ratio is, verifying the fifth hypothesis.

Despite low compressibility of floating-point data using general-purpose compression techniques, there are several proposals to improve their compressibility. These techniques (Burtscher 


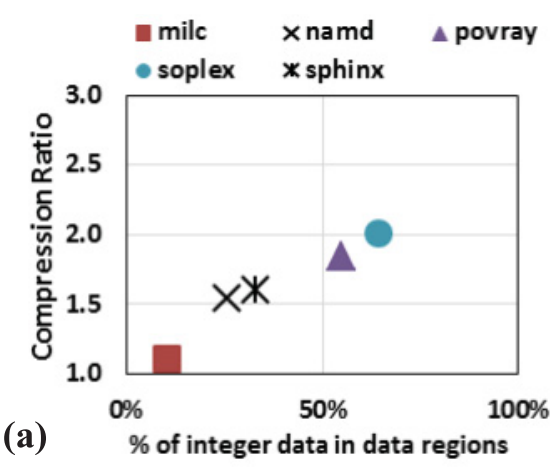

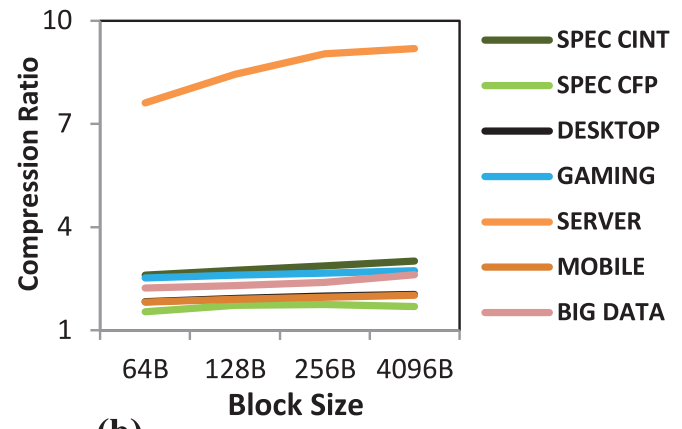

(b)

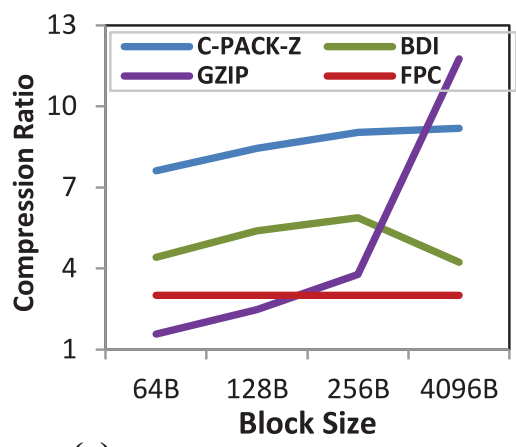

(c)

Fig. 6. (a) Percentage of integer blocks in data regions of memory vs. compression ratio of SPEC CFP using C-PACK + Z. (b) Memory compression with different block sizes for all domains (c) for servers with different algorithms.

et al. 2010, 2007) exploit the low entropy (Mahapatra et al. 2003, 2005) and similarities among a sequence of floating-point values to compress them.

\subsection{Compressibility of Different Data Types: Instructions versus Data}

Existing general-purpose compression algorithms usually achieve good compressibility for data blocks with repeated bit patterns. However, instruction blocks are mostly considered as uncompressible using general-purpose compression algorithms (Kant et al. 2002).

In general, all our studied algorithms perform similarly and poorly in compressing instruction blocks. Overall, the compression ratio of instruction blocks is on average 1.13 using C-PACK $+\mathrm{Z}$. Over $70 \%$ of instruction blocks are uncompressible (i.e., compressed block size of 64B) or poorly compressible (i.e., compressed block size $\geq 48 \mathrm{~B}$ ) for our workloads. We observe similar results with other compression algorithms. Since instruction blocks have more complicated coding and patterns than data blocks, general-purpose algorithms fail to benefit them, verifying the sixth hypothesis.

There are several specialized techniques to improve compressibility of instructions (Beszédes et al. 2003). These techniques mostly exploit the low entropy among a series of instructions (Mahapatra et al. 2003, 2005), find frequently used instruction sequences in instruction stream, and replace those with small code-words (Lefurgy et al. 1997; Chen al. 1997; Jin et al. 2000). 


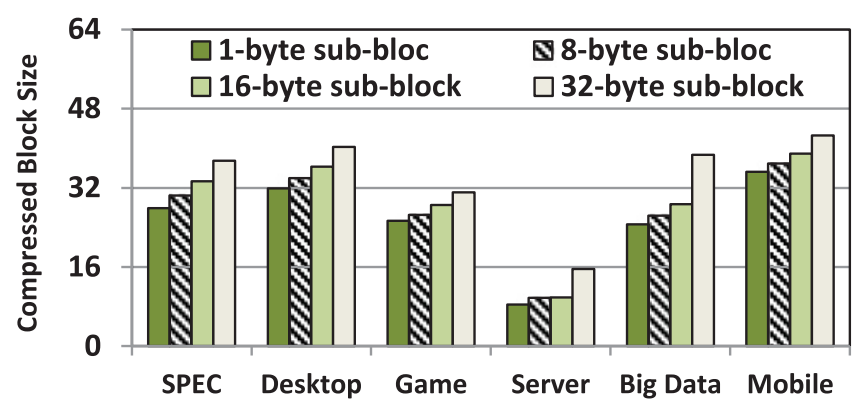

Fig. 7. Impact of sub-blocking on compressed size.

\subsection{How Sensitive Is Compressibility to Block Granularity?}

Conventional belief is that larger blocks are more compressible (Mahapatra et al. 2003, 2005; Sardashti and Wood 2013). In general, more redundancy is present within larger blocks, but more effort is also needed to compress them. Figure 6(b) shows the compression ratio of our workloads in main memory using the C-PACK $+Z$ algorithm at different granularities (64B to 4096B). Some workloads, such as servers, have significantly higher compression ratio (i.e., 20\%) at page granularity, while compression ratio of others (e.g., mobile) would not change as much.

Sensitivity to block size also depends on compression algorithm. As shown in Figure 6(c), while compressibility of servers improves at larger block sizes with $\mathrm{C}-\mathrm{PACK}+\mathrm{Z}$, larger block sizes do not impact compressibility with FPC $+\mathrm{Z}$ and even can hurt efficiency of BDI. FPC $+\mathrm{Z}$ compresses each word in a block separately, so it is not sensitive to block size. The efficiency of BDI reduces at page granularity as it would be hard to find a small number of base values that are good for all words in a page.

On the other hand, gzip is highly sensitive to block size. When applied at page granularity, gzip can provide on average $4.5 \times$, and up to $12 \times$ (for Fileserver) higher compression ratio for servers. Overall, compressibility is not a direct function of block size. Compression sensitivity to block size depends on compression algorithm and data being compressed.

Note that although using larger blocks might improve compression ratio for some workloads, the benefits come at higher costs. Using larger blocks might negate the benefits from compression by increasing cache pollution, reducing cache efficiency, and incurring energy overheads (Yoon et al. 2011).

\subsection{Would Sub-Blocking Eliminate Fragmentation?}

While the compression algorithm determines how many bits are needed to represent the information in a cache block, a compaction mechanism is necessary to store multiple compressed blocks in the cache. To avoid internal fragmentation (wasted space in blocks), an ideal compaction mechanism would store compressed blocks at byte (or even bit) granularity; however, this incurs impractically high overheads on cache/memory metadata (Sardashti and Wood 2013). Early works store a compressed block as half a block if possible otherwise they store it as uncompressed. Blocks that compress to far fewer bits than half a cache block result in high internal fragmentation. Alternatively some proposals store compressed blocks as a variable number of small sub-blocks; this helps reduce internal fragmentation while keeping the overheads low (Alameldeen and Wood 2004; Sardashti and Wood 2013; Sardashti et al. 2014).

To study the potential effectiveness of sub-blocking, Figure 7 shows the average compressed block size when using different sub-block sizes. The larger the compressed block size, 
the lower the effective capacity. Using only 32-byte sub-blocks (i.e., compressing a block to half if possible) would increase the average compressed size by $33 \%$ and up to 2 times (for libquantum).

This means a roughly $33 \%$ increase in internal fragmentation on average. However, using small sub-block sizes, we can retain some of the benefits of byte-granularity with significantly lower overheads. Using eight-block sub-blocks would increase the average compressed size by only $7 \%$ compared to 1-byte sub-blocks, while it significantly lowers internal fragmentation compared to 32-byte sub-blocks. Overall, storing blocks as small sub-blocks reduces internal fragmentation, indicating the importance of designs that enable sub-blocking.

\section{SUMMARY AND DISCUSSION}

\subsection{Related Work}

Data compression has been widely used to increase storage capacity or improve network bandwidth. Sardashti et al. (2015) and Mittal et al. (2016) summarize several of these techniques.

There are many proposals on using compression for improving main memory efficiency in terms of bandwidth and storage. IBM MXT (Abali et al. 2001) uses main memory compression to effectively double the main memory capacity. Ekman et al. (2005) propose a compressed memory design that extends page table entries to keep compressed block sizes. The decoupled zero-compressed memory (Dusser et al. 2011) detects null blocks using a design based on decoupled sectored setassociative caches. Pekhimenko et al. (2013) propose a new compressed memory design that exploits compression locality to simplify address mapping.

Unlike these techniques that target improving memory space, some others focus on bandwidth utilization. MemZip (Shafiee et al. 2014) keeps the data in each memory block compressed but only for the purpose of saving bandwidth, not for the purpose of gaining more memory capacity. They save performance and energy by saving bandwidth and generating fewer channel requests on memory accesses. Kim et al. (2016) also proposes a new compression to improve memory bandwidth.

Several previous works focus on cache compression. SC ${ }^{2}$ (Arelakis et al. 2014) utilizes statistical compression to improve cache performance and power. Lee's proposed compressed cache (Lee et al. 2000) uses a direct tag-data mapping, packing adjacent, aligned blocks into a single line if both blocks are compressed by at least a factor of two. Alameldeen and Wood (2004) propose a variablesize compressed cache that uses extra metadata (block size) to locate a block. They also propose an adaptive technique to turn off compression whenever not beneficial, which could potentially be used with SCC. Similarly, IIC-C (Hallnor et al. 2005) supports variable compressed blocks but uses forward pointers to associate a tag with its sub-blocks resulting in high cache area overhead. Dusser et al. (2011) augment the conventional cache with a specialized cache to store zero blocks (ZCA). DCC (Sardashti and Wood 2013), SCC (Sardashti et al. 2014), and YACC (Sardashti et al. 2016) track super-blocks to track more blocks with low area overhead. More recently, DISH (Panda et al. 2016) addresses some of the compaction problems with SCC and YACC by dictionary sharing among neighboring blocks.

Compression has also been used to reduce cache power consumption. Residue cache (Kim et al. 2011) reduces L2 cache area and power by compressing and storing blocks in half size if compressible. DZC (Villa et al. 2000) also reduces power by only storing non-zero bytes of cache blocks. Similarly, FVC (Yang et al. 2000; Yang et al. 2002) and significance compression (Kim et al. 2002) can reduce power by accessing half a block if it is compressible. Similarly, DCC, SCC, and YACC can also reduce LLC dynamic power due to accessing fewer bytes for compressed blocks. 
S. Baek et al. (2013) improves the performance of compressed caches using a size-aware cache replacement mechanism. Pekhimenko et al. (2015) similarly propose tailored replacement and insertion policies for compressed caches. Base-Victim (Gaur et al. 2016) also target lowering the negative impacts of compression on replacement.

Several recent proposals consider compression for other domains. There are proposal exploiting compression in GPUs (Pekhimenko et al. 2016; Vijaykumar 2015; Lee et al. 2015). DICE (Young et al. 2017) applies compression to DRAM caches. Palangappa et al. (Palangappa et al. 2016, 2017) use compression to improve efficiency of NVM memories. MORC (Nguyen et al. 2015) proposes a log-based compressed cache for throughput-oriented applications.

\subsection{Contributions}

Many proposals on compression rely on workload properties that have only been demonstrated to hold for short simulated runtime of small benchmarks with mostly synthetic data. In this article, our goal was to determine whether these workload properties hold for a wide range of workloads from different domains, including both real applications running with real data and benchmarks running to completion, on real machines.

To enable studying compression on real machines, we have developed two new tools to study cache and memory compression on real machines. Using our tools, we find several insights. We showed that workloads from different domains exhibit different compressibility, with servers as the highest compressible, and mobile benchmarks as the least compressible. In fact, we showed that real applications tend to have significantly higher compressibility than benchmarks, with average compression ratios of 3.7 versus 2.3 . SPEC benchmarks, on the other hand, are generally representative of real desktop applications.

Despite such differences, we showed that overall compression could be of general use both in caches and main memory with more than $50 \%$ of the workloads being compressible by at least a factor of two. Memory data are generally more compressible than cache data due to abundance of zero blocks.

By testing basic hypotheses that have formed the foundation for many compressed cache/memory designs we implicitly tested whether those designs would work in a real machine. We showed that many of the workloads behaviors that have been shown to hold for small samples of benchmarks actually hold for large, real applications. This suggests that real applications could also achieve performance, energy, or area benefits of proposed compression mechanisms.

Given the widespread compressibility of data, designers can leverage compression to improve effective capacity of caches or memory in memory intensive systems, such as servers or desktop (Sardashti and Wood 2013), to improve bandwidth and communication cost in GPU systems (Sathish et al. 2012), or to reduce storage size in area-constrained mobile systems (Kim et al. 2011) or big data systems with low sensitivity to caches (Ferdman et al. 2012).

\subsection{Were We on the Right Path in the Past?}

We demonstrated that many compression behaviors hold quite broadly, not just for small samples but across complicated workloads. But there is also not just one behavior. When using benchmarks, we showed that it is important to run them to completion, as compressibility changes over time. As another example, we discussed in Section 3.4, unlike SPEC benchmarks, not all real applications have high compression locality. Thus, it is critical for designers to study wider range of workloads. Given the emergence of new application areas, creating representative benchmarks and simulating them would become a burden. The mechanisms proposed in this article could help researchers to study a wide range of new applications with a lot less tedious effort. 


\section{REFERENCES}

B. Abali, H. Franke, X. Shen, D. Poff, and T. Smith. 2001. Performance of hardware compressed main memory. In Proceedings of the 7th IEEE Symposium on High-Performance Computer Architecture.

Alaa R. Alameldeen and David A. Wood. 2004. Adaptive cache compression for high-performance processors. In Proceedings of the 31st Annual International Symposium on Computer Architecture (ISCA-31).

Apple OS X Mavericks. 2013. Retrieved from http://www.apple.com/media/us/osx/2013/docs/OSX_Mavericks_Core_ Technology_Overview.pdf.

Angelos Arelakis and P. Stenstrom. 2014. Sc2: A statistical compression cache scheme. In Proceeding of the 41st Annual International Symposium on Computer Architecuture (ISCA'14). IEEE Press, 145-156.

Seungcheol Baek, Hyung Gyu Lee, Chrysostomos Nicopoulos, Junghee Lee, and Jongman Kim. 2013. ECM: Effective capacity maximizer for high-performance compressed caching. In Proceedings of IEEE Symposium on High-Performance Computer Architecture.

Á. Beszédes, R. Ferenc, T. Gyimóthy, A. Dolenc, and K. Karsisto. 2003. Survey of code-size reduction methods. ACM Comput. Surv. 35, 3 (2003), 223-267.

N. Binkert, B. Beckmann, G. Black, S. Reinhardt, A. Saidi, A. Basu, J. Hestness, D. Hower, T. Krishna, S. Sardashti, R. Sen, K. Sewell, M. Shoaib, N. Vaish, M. Hill, and D. Wood. 2011. The gem5 simulator. ACM SIGARCH Computer Architecture News. 1-7.

M. Burtscher and P. Ratanaworabhan. 2007. High throughput compression of double-precision floating-point data. Data Compression Conference.

M. Burtscher and P. Ratanaworabhan. 2010. gFPC: A self-tuning compression algorithm. In Proceedings of the Data Compression Conference.

I. Chen, P. Bird, and T. Mudge. 1997. The impact of instruction compression on I-cache performance. Tech. Rep. CSE-TR330-97, EECS Department, University of Michigan.

Xi Chen, Lei Yang, Robert P. Dick, Li Shang, and Haris Lekatsas. 2010. C-pack: A high-performance microprocessor cache compression algorithm. IEEE Transactions on Very Large Scale Integration (VLSI) Systems. 1196-1208.

Yann Collet and Chip Turner. 2016. Facebook zstandard compression: Smaller and faster data compression with zstandard. Retrieved from https://code.facebook.com/posts/1658392934479273/smaller-and-faster-data-compressionwith-zstandard/.

Coremark. Retrieved from www.coremark.org.

Arelakis F. Dahlgren and P. Stenstrom. 2015. Hycomp: A hybrid cache compression method for selection of data-typespecific compression methods. In Proceedings of the 48th International Symposium on Microarchitecture (MICRO-48). ACM, 38-49.

Julien Dusser and Andre Seznec. 2011. Decoupled zero-compressed memory. In Proceedings of the 6th International Conference on High Performance and Embedded Architectures and Compilers.

M. Ekman and P. Stenstrom. 2005. A robust main-memory compression scheme. In Proceedings of the 32nd Annual International Symposium on Computer Architecture. 74-85.

E. Hallnor and S. Reinhardt. 2005. A unified compressed memory hierarchy. In Proceedings of the 11th International Symposium on High-Performance Computer Architecture.

M. Ferdman, A. Adileh, O. Kocberber, S. Volos, M. Alisafaee, D. Jevdjic, C. Kaynak, A. Popescu, A. Ailamaki, and B. Falsafi. 2012. Clearing the clouds: A study of emerging scale-out workloads on modern hardware. In Proceedings of the 17th International Conference on Architectural Support for Programming Languages and Operating Systems (ASPLOS'12).

J. Gandhi, A. Basu, M. Hill, and M. Swift 2014. BadgerTrap: A tool to instrument x86-64 TLB misses. SIGARCH Computer Architecture News (CAN), 2014

Jayesh Gaur, Alaa R. Alameldeen, and Sreenivas Subramoney. 2016. Base-victim compression: An opportunistic cache compression architecture. In Proceedings of the 43th Annual International Symposium on Computer Architecture (ISCA'16).

R. C. Murphy, K. B. Wheeler, B. W. Barrett, and J. A. Ang. 2010. Introducing the Graph 500. Cray User Group 2010 Proceedings.

A. Gutierrez, R. Dreslinski, T. Wenisch, T. Mudge, A. Saidi, C. Emmons, and N. Paver. 2011. Full-system analysis and characterization of interactive smartphone applications. In Proceedings of the IEEE International Symposium on Workload Characterization (IISWC'11).

G. Hamerly, E. Perelman, J. Lau, and B. Calder. 2005. SimPoint 3.0: Faster and more flexible program analysis. In Proceedings of the Workshop on Modeling, Benchmarking and Simulation.

Y. Jin and R. Chen 2000. Instruction Cache Compression for Embedded Systems. Berkley Wireless Research Center,” Technical Report, 2000.

K. Kant and R. Iyer. 2002. Compressibility characteristics of address/data transfers in commercial workloads. In Proceedings of the 5th Workshop on Computer Architecture Evaluation Using Commercial Workloads. 59-67. 
Nam Sung Kim, Todd Austin, and Trevor Mudge. 2002. Low-energy data cache using sign compression and cache line bisection. In Proceedings of the 2nd Annual Workshop on Memory Performance Issues.

Soontae Kim, Jesung Kim, Jongmin Lee, and Seokin Hong. 2011. Residue cache: A low-energy low-area L2 cache architecture via compression and partial hits. In Proceedings of the 44th Annual IEEE/ACM International Symposium on Microarchitecture.

Jungrae Kim, Michael Sullivan, Esha Choukse, and Mattan Erez. 2016. Bit-plane compression: Transforming data for better compression in many-core architectures. In Proceedings of the 43th Annual International Symposium on Computer Architecture (ISCA'16)

Jang-Soo Lee, Won-Kee Hong, and Shin-Dug Kim. 2000. An on-chip cache compression technique to reduce decompression overhead and design complexity. Journal of Systems Architecture: The EUROMICRO fournal 46, 15 (2000), 1365-1382. 2000 .

Sangpil Lee, Keunsoo Kim, Gunjae Koo, Hyeran Jeon, Won Woo Ro, and Murali Annavaram. 2015. Warped-compression: Enabling power efficient GPUs through register compression. In Proceedings of the 44th Annual International Symposium on Computer Architecture (ISCA'15).

C. Lefurgy, P. Bird, I. Chen, and T. Mudge. 1997. Improving code density using compression techniques. In Proceedings of the 30th Annual ACM/IEEE International Symposium on Microarchitecture. 194-203.

N. R. Mahapatra, J. Liu, K. Sundaresan, S. Dangeti, and B. V. Venkatrao. 2003. The potential of compression to improve memory system performance, power consumption, and cost. In Proceedings of IEEE Performance, Computing and Communications Conference.

N. R. Mahapatra, J. Liu, K. Sundaresan, S. Dangeti, and B. V. Venkatrao 2005. A limit study on the potential of compression for improving memory system performance, power consumption, and cost. F. Instruct.-Level Parallel. 7 (2005), 1-37.

Sparsh Mittal and Jeffrey S. Vetter. 2016. A survey of architectural approaches for data compression in cache and main memory systems. IEEE Transactions on Parallel and Distributed Systems, 2016.

Tri M. Nguyen and David Wentzlaff. 2015. MORC: A manycore-oriented compressed cache. In Proceedings of the IEEE/ACM International Symposium on Microarchitecture (MICRO'15).

Poovaiah M. Palangappa and Kartik Mohanram. 2016. CompEx: Compression-expansion coding for energy, latency, and lifetime improvements in MLC/TLC NVM. In Proceedings of the IEEE International Symposium on High Performance Computer Architecture (HPCA'16).

Poovaiah M. Palangappa and Kartik Mohanram. 2017, CompEx++: Compression-expansion coding for energy, latency, and lifetime improvements in MLC/TLC NVMs. ACM Transactions on Architecture and Code Optimization (TACO), 2017.

Biswabandan Panda (INRIA) and André Seznec. 2016. Dictionary sharing: An efficient cache compression scheme for compressed caches. In Proceedings of the Annual IEEE/ACM International Symposium on Microarchitecture, 2016.

Gennady Pekhimenko, Evgeny Bolotin, Nandita Vijaykumar, Onur Mutlu, Todd C. Mowry, Stephen W. Keckler. 2016. A case for toggle-aware compression for GPU systems. In Proceedings of the IEEE International Symposium on High Performance Computer Architecture (HPCA'16), 2016.

G. Pekhimenko, T. Huberty, R. Cai, O. Mutlu, P. B. Gibbons, M. A. Kozuch, and T. C. Mowry. 2015. Exploiting compressed block size as an indicator of future reuse. In Proceedings of the 2015 IEEE 21st International Symposium on High Performance Computer Architecture (HPCA'15). 51-63.

Gennady Pekhimenko, Vivek Seshadri, Onur Mutlu, Phillip B. Gibbons, Michael A. Kozuch, and Todd C. Mowry. 2012. Basedelta-immediate compression: Practical data compression for on-chip caches. In Proceedings of the 21st International Conference on Parallel Architectures and Compilation Techniques (PACT'12). ACM, New York, NY, 377-388.

Gennady Pekhimenko, Vivek Seshadri, Yoongu Kim, Hongyi Xin, Onur Mutlu, Phillip B. Gibbons, Michael A. Kozuch, and Todd C. Mowry. 2013. Linearly compressed pages: A low-complexity, low-latency main memory compression framework. In Proceedings of the Annual IEEE/ACM International Symposium on Microarchitecture, 2013.

P. Ratanaworabhan, J. Ke, and M. Burtscher. 2006. Fast lossless compression of scientific floating-point data. In Proceedings of the Data Compression Conference.

Somayeh Sardashti and David A. Wood. 2013. Decoupled compressed cache: Exploiting spatial locality for energy-optimized compressed caching. In Proceedings of the Annual IEEE/ACM International Symposium on Microarchitecture.

Somayeh Sardashti, Angelos Arelakis, Per Stenstrom, and David A. Wood. 2015. A primer on compression in the memory hierarchy. Morgan and Claypool.

Somayeh Sardashti, Andre Seznec, and David A. Wood. 2014. Skewed compressed caches. In Proceedings of the 47th Annual IEEE/ACM International Symposium on Microarchitecture (MICRO-47).

Somayeh Sardashti, Andre Seznec, and David A. Wood. 2016. Yet another compressed cache: A low-cost yet effective compressed cache. ACM Transactions on Architecture and Code Optimization (TACO), 2016.

Vijay Sathish, Michael J. Schulte, and Nam Sung Kim. 2012. Lossless and lossy memory I/O link compression for improving performance of GPGPU workloads. In Proceedings of the 21st International Conference on Parallel Architectures and Compilation Techniques. 
Ali Shafiee, Meysam Taassori, Rajeev Balasubramonian, and Al Davis. 2014. Memzip: Exploiting unconventional benefits from memory compression. In Proceedings of the IEEE International Symposium on High Performance Computer Architecture (HPCA'14).

Luis Villa, Michael Zhang, and Krste Asanovic. 2000. Dynamic zero compression for cache energy reduction. In Proceedings of the 33rd Annual ACM/IEEE International Symposium on Microarchitecture.

Nandita Vijaykumar, Gennady Pekhimenko, Adwait Jog, Abhishek Bhowmick, Rachata Ausavarungnirun, Chita Das, Mahmut Kandemir, Todd C. Mowry, and Onur Mutlu. 2015. A case for core-assisted bottleneck acceleration in GPUs: Enabling flexible data compression with assist warps. In Proceedings of the 44th Annual International Symposium on Computer Architecture (ISCA'15).

C. Wu, A. Jaleel, W. Hasenplaugh, M. Martonosi, S. Steely, and J. Emer. 2011. SHiP: Signature-based hit predictor for high performance caching. In Proceedings of the 44th Annual IEEE/ACM International Symposium on Microarchitecture.

Jun Yang and Rajiv Gupta. 2002. Frequent value locality and its applications. ACM Trans. Embed. Comput. Syst. 2002.

Jun Yang, Youtao Zhang, and Rajiv Gupta. 2000. Frequent value compression in data caches. In Proceedings of the IEEE/ACM International Symposium on Microarchitecture (MICRO'00).

D. Yoon, M. Jeong, and M. Erez. 2011. Adaptive granularity memory systems: A tradeoff between storage efficiency and throughput. In Proceeding of the 38th Annual International Symposium on Computer Architecture.

Vinson Young, Prashant J. Nair, Moinuddin K. Qureshi. 2017. DICE: Compressing DRAM Caches for bandwidth and capacity. In Proceedings of the 44th Annual International Symposium on Computer Architecture (ISCA'17).

Received June 2016; revised July 2017; accepted September 2017 